CUBO A Mathematical Journal Vol.12, № 03, (153-165). October 2010

\title{
Existence of Periodic Solutions for a Class of Second-Order Neutral Differential Equations with Multiple Deviating Arguments ${ }^{1}$
}

\author{
ChenguUn Guo \\ School of Applied Mathematics, Guangdong, \\ University of Technology 510006, P.R.China \\ email: guochj817@163.com \\ DONAL O'REGAN \\ Department of mathematics, National University of Ireland, \\ Galway, Ireland \\ email: donal.oregan@nuigalway.ie \\ AND \\ RAVi P. Agarwal \\ Department of Mathematical Sciences, Florida Institute of Technology, \\ Melbourne, Florida 32901, USA \\ email: agarwal@fit.edu
}

\begin{abstract}
Using Kranoselskii fixed point theorem and Mawhin's continuation theorem we establish the existence of periodic solutions for a second order neutral differential equation with multiple deviating arguments.
\end{abstract}

\footnotetext{
${ }^{1}$ This project is supported by grant 10871213 from NNSF of China, by grant 06021578 from NSF of Guangdong.
} 


\section{RESUMEN}

Usando el teorema del punto fijo de Kranoselskii y el teorema de continuación de Mawhin establecemos la existencia de soluciones periódicas de una ecuación diferencial neutral de segundo orden con argumento de desviación multiple.

Key words and phrases: Periodic solution, Multiple deviating arguments, Neutral differential equation, Kranoselskii fixed point theorem, Mawhin's continuation theorem.

Math. Subj. Class.: 34K15; 34C25.

\section{Introduction}

In this paper, we discuss the second-order neutral differential equation with multiple deviating arguments of the form

$$
x^{\prime \prime}(t)+c x^{\prime \prime}(t-\tau)+a(t) x(t)+g\left(t, x\left(t-\tau_{1}(t)\right), x\left(t-\tau_{2}(t)\right) \cdots, x\left(t-\tau_{n}(t)\right)\right)=p(t),
$$

where $|c|<1, \tau$ is a constant, $\tau_{i}(t)(i=1,2, \cdots, n), a(t)$ and $p(t)$ are real continuous functions defined on $\mathbf{R}$ with positive period $T$ and $g\left(t, x_{1}, x_{2}, \cdots, x_{n}\right) \in C(\mathbf{R} \times \mathbf{R} \times \mathbf{R} \times \cdots \times \mathbf{R}, \mathbf{R})$ and is $T$-periodic in $t$.

Periodic solutions for differential equations were studied in $[2-4,6-10,12,15]$ and we note that most of the results in the literatue concern delay problems. There are only a few papers $[1,5,11,13,14]$ which discuss neutral problems.

For the sake of completeness, we first state Kranoselskii fixed point theorem and Mawhin's continuation theorem [3].

Theorem A (Kranoselskii). Suppose that $\Omega$ is a Banach space and $X$ is a bounded, convex and closed subset of $\Omega$. Let $U, S: X \rightarrow \Omega$ satisfy the following conditions:

(1) $U x+S y \in X$ for any $x, y \in X$;

(2) $U$ is a contraction mapping;

(3) $S$ is completely continuous.

Then $U+S$ has a fixed point in $X$.

Let $\mathrm{X}$ and $\mathrm{Y}$ be two Banach space and $L: D o m L \subset X \longrightarrow Y$ is a linear mapping and $N: X \longrightarrow Y$ is a continuous mapping. The mapping $\mathrm{L}$ will be called a Fredholm mapping of index zero if $\operatorname{dimKerL}=$ codimImL $<+\infty$, and $\operatorname{ImL}$ is closed in Y. If $\mathrm{L}$ is a Fredholm mapping of index zero, there exist continuous projectors $P: X \longrightarrow X$ and $Q: Y \longrightarrow Y$ such that 
$\operatorname{Im} P=\operatorname{Ker} L$ and $\operatorname{Im} L=K e r Q=\operatorname{Im}(I-Q)$. It follows that $\left.L\right|_{D o m L \cap K e r P}:(I-P) X \longrightarrow \operatorname{ImL}$ has an inverse which will be denoted by $K_{P}$. If $\Omega$ is an open and bounded subset of X, the mapping $N$ will be called $L$-compact on $\Omega$ if $Q N(\bar{\Omega})$ is bounded and $\overline{K_{P}(I-Q) N(\bar{\Omega})}$ is compact. Since $\operatorname{ImQ}$ is isomorphic to $K e r L$, there exists an isomorphism $J: \operatorname{ImQ} \longrightarrow K e r L$.

Theorem B (Mawhin's continuation theorem[3]). Let L be a Fredholm mapping of index zero, and let $\mathrm{N}$ be $L$-compact on $\bar{\Omega}$. Suppose

(1) for each $\lambda \in(0,1)$ and $x \in \partial \Omega, L x \neq \lambda N x$ and

(2) for each $x \in \partial \Omega \cap \operatorname{Ker}(L), Q N x \neq 0$ and $\operatorname{deg}(Q N, \Omega \cap \operatorname{Ker}(L), 0) \neq 0$.

Then the equation $L x=N x$ has at least one solution in $\bar{\Omega} \cap D(L)$.

\section{Main Results}

Now we make the following assumption on $a(t)$ :

$\left(H_{1}\right) \quad\left(\frac{\pi}{T}\right)^{2}>M=\max _{t \in[0, T]} a(t) \geq a(t) \geq m=\min _{t \in[0, T]} a(t)>0$.

Our main results are the following theorems.

Theorem 2.1 Suppose $\left(H_{1}\right)$ holds and also assume there exists a constant $K_{1}>0$ such that $\left(H_{2}\right)$

$$
\|g\|_{0} \leq m-3|c| M-\|p\|_{0},
$$

where $\|g\|_{0}=\max _{\left\{t \in[0, T],\left|x_{1}\right| \leq K_{1}, \cdots,\left|x_{n}\right| \leq K_{1}\right\}}\left|g\left(t, x_{1}, x_{2}, \cdots, x_{n}\right)\right|$ and $\|p\|_{0}=\max _{t \in[0, T]}|p(t)|$.

Then Eq.(1.1) possesses a nontrivial $T$-periodic solution.

Theorem 2.2 Suppose $\left(H_{1}\right)$ holds and also assume

$\left(H_{3}\right)$

$$
\left|g\left(t, x_{1}, x_{2}, \cdots, x_{n}\right)\right| \leq \gamma \sum_{i=1}^{n}\left|x_{i}\right| .
$$

Then Eq.(1.1) has at least one $T$-periodic solution as $0<\gamma<\frac{1}{n}[(1-|c|) m-|c| M]$.

In order to prove the main theorems we need some preliminaries. Set

$$
X:=\left\{x \mid x \in C^{2}(\mathbf{R}, \mathbf{R}), x(t+T)=x(t), \forall t \in \mathbf{R}\right\}
$$

and $x^{(0)}(t)=x(t)$ and define the norm on $X$ as follows

$$
\|x\|=\max _{t \in[0, T]}|x(t)|+\max _{t \in[0, T]}\left|x^{\prime}(t)\right|+\max _{t \in[0, T]}\left|x^{\prime \prime}(t)\right| .
$$


Remark 2.3 If $x \in X$, then it follows that $x^{(i)}(0)=x^{(i)}(T)(i=0,1,2)$.

In order to prove our main results, we need the following Lemma [10].

Lemma 2.4 ([10]). Suppose that $M$ is a positive number and satisfies $0<M<\left(\frac{\pi}{T}\right)^{2}$. Then for any function $\varphi$ defined in $[0, T]$, the following equation

$$
\left\{\begin{array}{l}
x^{\prime \prime}(t)+M x(t)=\varphi(t), \\
x(0)=x(T), x^{\prime}(0)=x^{\prime}(T)
\end{array}\right.
$$

has a unique solution

$$
x(t)=\int_{0}^{T} G(t, s) \varphi(s) d s
$$

where

$$
G(t, s)\left\{\begin{array}{c}
w(t-s), \quad(k-1) T \leq s \leq t \leq k T \\
w(T+t-s), \quad(k-1) T \leq t \leq s \leq k T(k \in \mathbf{N}), \\
w(t)=\frac{\cos \alpha\left(t-\frac{T}{2}\right)}{2 \alpha \sin \frac{\alpha T}{2}}
\end{array}\right.
$$

and $\alpha=\sqrt{M}$. Here

$$
\max _{t \in[0, T]} \int_{0}^{T}|G(t, s)| d s=\frac{1}{M}
$$

Proof of Theorem 2.1: For $\forall x \in X$, define the operators $U: X \longrightarrow X$ and $S: X \longrightarrow X$ respectively by

$$
(U x)(t)=-c x(t-\tau)
$$

and

$$
\begin{aligned}
(S x)(t) & =c x(t-\tau)+\int_{0}^{T} G(t, s)\left[-c x^{\prime \prime}(s-\tau)(M-a(s)) x(s)+p(s)\right. \\
& \left.-g\left(s, x\left(s-\tau_{1}(s)\right), x\left(s-\tau_{2}(s)\right) \cdots, x\left(s-\tau_{n}(s)\right)\right)\right] d s .
\end{aligned}
$$

It is clear that a fixed point of $U+S$ is a $T$-periodic solution of Eq.(1.1).

We are going to demonstrate that $U$ and $S$ satisfy the conditions of Theorem A.

Let $x, y \in X$ and $|x| \leq K_{1},|y| \leq K_{1}$ (here $K_{1}$ is as in the statement of Theorem 2.1). Now we prove that $|U x+S y| \leq K_{1}$ holds.

First, we have the following equality:

$$
\int_{0}^{T} G(t, s) x^{\prime \prime}(s-\tau) d s=M \int_{0}^{T} G(t, s) x(s-\tau) d s .
$$


In fact, we have from Lemma 2.4

$$
\begin{aligned}
\int_{0}^{T} G(t, s) x^{\prime \prime}(s-\tau) d s & =\int_{0}^{t} \frac{\cos \alpha\left(t-s-\frac{T}{2}\right)}{2 \alpha \sin \frac{T \alpha}{2}} d\left[x^{\prime}(s-\tau)\right]+\int_{t}^{T} \frac{\cos \alpha\left(t-s+\frac{T}{2}\right)}{2 \alpha \sin \frac{T \alpha}{2}} d\left[x^{\prime}(s-\tau)\right] \\
& =\left.\frac{\cos \alpha\left(t-s-\frac{T}{2}\right)}{2 \alpha \sin \frac{T \alpha}{2}} x^{\prime}(s-\tau)\right|_{0} ^{t}-\alpha \int_{0}^{t} \frac{\sin \alpha\left(t-s-\frac{T}{2}\right)}{2 \alpha \sin \frac{T \alpha}{2}} d[x(s-\tau)] \\
& +\left.\frac{\cos \alpha\left(t-s+\frac{T}{2}\right)}{2 \alpha \sin \frac{T \alpha}{2}} x^{\prime}(s-\tau)\right|_{t} ^{T}-\alpha \int_{t}^{T} \frac{\sin \alpha\left(t-s+\frac{T}{2}\right)}{2 \alpha \sin \frac{T \alpha}{2}} d[x(s-\tau)] \\
& =-\alpha\left[\left.\frac{\sin \alpha\left(t-s-\frac{T}{2}\right)}{2 \alpha \sin \frac{T \alpha}{2}} x(s-\tau)\right|_{0} ^{t}+\left.\frac{\sin \alpha\left(t-s+\frac{T}{2}\right)}{2 \alpha \sin \frac{T \alpha}{2}} x(s-\tau)\right|_{t} ^{T}\right] \\
& +\alpha^{2}\left[\int_{0}^{t} \frac{\cos \alpha\left(t-s-\frac{T}{2}\right)}{2 \alpha \sin \frac{T \alpha}{2}} x(s-\tau) d s+\int_{t}^{T} \frac{\cos \alpha\left(t-s+\frac{T}{2}\right)}{2 \alpha \sin \frac{T \alpha}{2}} x(s-\tau) d s\right] \\
& =M \int_{0}^{T} G(t, s) x(s-\tau) d s,
\end{aligned}
$$

so (2.3) holds.

From $\left(H_{1}\right),\left(H_{2}\right)$ and (2.1)-(2.3), we have

$$
\begin{aligned}
|(U y)(t)+(S x)(t)| & \leq|(U y)(t)|+|(S x)(t)| \\
& \leq 2|c| K_{1}+\mid \int_{0}^{T} G(t, s)(M-a(s)) x(s)-c x^{\prime \prime}(s-\tau)+p(s) \\
& \left.-g\left(s, x\left(s-\tau_{1}(s)\right), x\left(s-\tau_{2}(s)\right) \cdots, x\left(s-\tau_{n}(s)\right)\right)\right] d s|+| c \mid K_{1} \\
& \leq 2|c| K_{1}+\frac{M-m}{M} K_{1}+\frac{\|g\|_{0}}{M}+|c| M \| \int_{0}^{T} G(t, s) x(s-\tau) d s \mid \\
& \leq 3|c| K_{1}+\frac{M-m}{M} K_{1}+\frac{\|g\|_{0}+\|p\|_{0}}{M} \\
& \leq K_{1}, \quad x, y \in X
\end{aligned}
$$

where $\|g\|_{0}$ and $\|p\|_{0}$ are given in $\left(H_{2}\right)$.

Set

$$
K_{2}=\frac{\rho_{0}\left[(M-m) K_{1}+|c| K_{3}+\|g\|_{0}+\|p\|_{0}\right]}{1-2|c|},
$$

where $\rho_{0}=\frac{T}{2 \sin \frac{T \alpha}{2}}$,

$$
K_{3}=\frac{M K_{1}+\|g\|_{0}+\|p\|_{0}}{1-|c|}
$$

and

$$
G=\left\{x \in X:|x(t)| \leq K_{1},\left|x^{\prime}(t)\right| \leq K_{2},\left|x^{\prime \prime}(t)\right| \leq K_{3}\right\} .
$$

It is clear that $G$ is a bounded, convex and closed subset of $X$.

(1) For $\forall x, y \in G$, we will show that

$$
\left|\frac{d}{d t}[(U y)(t)+(S x)(t)]\right| \leq K_{2}
$$


and

$$
\left|\frac{d^{2}[(U y)(t)+(S x)(t)]}{d t^{2}}\right| \leq K_{3} .
$$

From (2.1) we have

$$
\frac{d}{d t}[(U x)(t)]=-c x^{\prime}(t-\tau)
$$

and

$$
\frac{d^{2}[(U x)(t)]}{d t^{2}}=-c x^{\prime \prime}(t-\tau) .
$$

Also from Lemma 2.4 and (2.2) we have

$$
\begin{aligned}
\frac{d}{d t}[(S x)(t)] & =\int_{0}^{T} G_{t}(t, s)\left[(M-a(s)) x(s)-c x^{\prime \prime}(s-\tau)+p(s)\right. \\
& \left.-g\left(s, x\left(s-\tau_{1}(s)\right), x\left(s-\tau_{2}(s)\right) \cdots, x\left(s-\tau_{n}(s)\right)\right)\right] d s+c x^{\prime \prime}(t-\tau),
\end{aligned}
$$

where

$$
G_{t}(t, s)\left\{\begin{array}{l}
\widetilde{w}(t-s), \quad(k-1) T \leq s \leq t \leq k T \\
\widetilde{w}(T+t-s), \quad(k-1) T \leq t \leq s \leq k T(k \in \mathbf{N})
\end{array}\right.
$$

and

$$
\widetilde{w}(t)=\frac{\sin \alpha\left(t-\frac{T}{2}\right)}{2 \sin \frac{\alpha T}{2}},
$$

since

$$
\begin{aligned}
\frac{d}{d t}[(S x)(t)]= & \left\{\int _ { 0 } ^ { T } G _ { t } ( t , s ) \left[(M-a(s)) x(s)-c x^{\prime \prime}(s-\tau)+p(s)\right.\right. \\
& \left.\left.-g\left(s, x\left(s-\tau_{1}(s)\right), x\left(s-\tau_{2}(s)\right) \cdots, x\left(s-\tau_{n}(s)\right)\right)\right] d s+c x^{\prime \prime}(t-\tau)\right\}^{\prime} \\
& =\left\{\int _ { 0 } ^ { t } \frac { \operatorname { c o s } \alpha ( t - s - \frac { T } { 2 } ) } { 2 \alpha \operatorname { s i n } \frac { T \alpha } { 2 } } \left[(M-a(s)) x(s)-c x^{\prime \prime}(s-\tau)+p(s)\right.\right. \\
& \left.\left.-g\left(s, x\left(s-\tau_{1}(s)\right), x\left(s-\tau_{2}(s)\right) \cdots, x\left(s-\tau_{n}(s)\right)\right)\right] d s+c x^{\prime \prime}(t-\tau)\right\}^{\prime} \\
& +\left\{\int _ { t } ^ { s } \frac { \operatorname { c o s } \alpha ( t - s + \frac { T } { 2 } ) } { 2 \alpha \operatorname { s i n } \frac { T \alpha } { 2 } } \left[(M-a(s)) x(s)-c x^{\prime \prime}(s-\tau)+p(s)\right.\right. \\
& \left.\left.-g\left(s, x\left(s-\tau_{1}(s)\right), x\left(s-\tau_{2}(s)\right) \cdots, x\left(s-\tau_{n}(s)\right)\right)\right] d s+c x^{\prime \prime}(t-\tau)\right\}^{\prime} \\
& =\alpha\left\{\int _ { 0 } ^ { t } \frac { \operatorname { c o s } \alpha ( t - s - \frac { T } { 2 } ) } { 2 \alpha \operatorname { s i n } \frac { T \alpha } { 2 } } \left[(M-a(s)) x(s)-c x^{\prime \prime}(s-\tau)+p(s)\right.\right. \\
& \left.\left.-g\left(s, x\left(s-\tau_{1}(s)\right), x\left(s-\tau_{2}(s)\right) \cdots, x\left(s-\tau_{n}(s)\right)\right)\right] d s+c x^{\prime \prime}(t-\tau)\right\} \\
& +\alpha\left\{\int _ { t } ^ { s } \frac { \operatorname { c o s } \alpha ( t - s + \frac { T } { 2 } ) } { 2 \alpha \operatorname { s i n } \frac { T \alpha } { 2 } } \left[(M-a(s)) x(s)-c x^{\prime \prime}(s-\tau)+p(s)\right.\right. \\
& \left.\left.-g\left(s, x\left(s-\tau_{1}(s)\right), x\left(s-\tau_{2}(s)\right) \cdots, x\left(s-\tau_{n}(s)\right)\right)\right] d s+c x^{\prime \prime}(t-\tau)\right\} .
\end{aligned}
$$


Note

$$
\int_{0}^{T} \mid G_{t}\left(t, s \mid d s \leq \frac{T}{2 \sin \frac{\alpha T}{2}}=\rho_{0}\right.
$$

and

$$
\frac{d^{2}[(S x)(t)]}{d t^{2}}=p(t)-a(t) x(t)-g\left(t, x\left(t-\tau_{1}(t)\right), x\left(t-\tau_{2}(t)\right) \cdots, x\left(t-\tau_{n}(t)\right)\right) .
$$

From (2.6),(2.7) and (2.10)-(2.13), we have

$$
\begin{aligned}
\left|\frac{d}{d t}[(U y)(t)+(S x)(t)]\right| & \leq\left|\frac{d}{d t}[(U y)(t)]\right|+\left|\frac{d}{d t}[(S x)(t)]\right| \\
& \leq 2|c| K_{2}+\rho_{0}\left[(M-m) K_{1}+|c| K_{3}+\|g\|_{0}+\|p\|_{0}\right] \\
& \leq K_{2}
\end{aligned}
$$

and

$$
\begin{aligned}
\left|\frac{d^{2}[(U y)(t)+(S x)(t)]}{d t^{2}}\right| & =\mid(M-a(t)) x(t)-c y^{\prime \prime}(t-\tau)+p(t) \\
& -g\left(t, x\left(t-\tau_{1}(t)\right), x\left(t-\tau_{2}(t)\right) \cdots, x\left(t-\tau_{n}(t)\right)\right) \mid \\
& \leq(M-m) K_{1}+|c| K_{3}+\|g\|_{0}+\|p\|_{0} \\
& \leq K_{3} .
\end{aligned}
$$

From (2.5), (2.14) and (2.15), we have $U x+S y \in G$ for $\forall x, y \in G$.

(2) $U$ is a contraction mapping.

Let $x, y \in G$ and we from (2.1) that

$$
\begin{aligned}
\|U x-U y\| & =\max _{t \in[0, T]}|c x(t-\tau)-c y(t-\tau)|+\max _{t \in[0, T]}\left|c x^{\prime}(t-\tau)-c y^{\prime}(t-\tau)\right| \\
& +\max _{t \in[0, T]}\left|c x^{\prime \prime}(t-\tau)-c y^{\prime \prime}(t-\tau)\right| \\
& =|c|\left[\max _{t \in[0, T]}|x(t-\tau)-y(t-\tau)|+\max _{t \in[0, T]}\left|x^{\prime}(t-\tau)-y^{\prime}(t-\tau)\right|\right. \\
& \left.+\max _{t \in[0, T]}\left|x^{\prime \prime}(t-\tau)-y^{\prime \prime}(t-\tau)\right|\right] \\
& =|c|\|x-y\| .
\end{aligned}
$$

Since $|c|<1, U$ is a contraction mapping.

(3) $S$ is completely continuous.

We can obtain the continuity of $S$ from the continuity of $a(t), p(t)$ and $g\left(t, x\left(t-\tau_{1}(t)\right), x(t-\right.$ $\left.\left.\tau_{2}(t)\right) \cdots, x\left(t-\tau_{n}(t)\right)\right)$ for $t \in[0, T], x \in G$. In fact, suppose that $x_{k} \in G$ and $\left\|x_{k}-s\right\| \rightarrow 0$ as 
$k \rightarrow+\infty$. Since $G$ is closed convex subset of $X$, we have $x \in G$. Then

$$
\begin{aligned}
\left|S x_{k}-S x\right| & =c\left[x_{k}(t-\tau)-x(t-\tau)\right]+c\left[x_{k}(t-\tau)-x(t-\tau)\right] \\
& +\int_{0}^{T} G(t, s)\left\{(M-a(s))\left(x_{k}(s)-x(s)\right)-c\left[x_{k}^{\prime \prime}(s-\tau)-x^{\prime \prime}(s-\tau)\right]\right. \\
& -\left[g\left(s, x_{k}\left(s-\tau_{1}(s)\right), x_{k}\left(s-\tau_{2}(s)\right) \cdots, x_{k}\left(s-\tau_{n}(s)\right)\right)\right. \\
& \left.\left.-g\left(s, x\left(s-\tau_{1}(s)\right), x\left(s-\tau_{2}(s)\right) \cdots, x\left(s-\tau_{n}(s)\right)\right)\right]\right\} d s .
\end{aligned}
$$

Using the Lebesgue dominated convergence theorem, we have from (2.12), (2.13) and (2.16) that

$$
\lim _{k \rightarrow+\infty}\left\|S x_{k}-S x\right\|=0 .
$$

Then $S$ is continuous.

Next, we prove that $S x$ is relatively compact. It suffices to show that the family of functions $\{S x: x \in G\}$ is uniformly bounded and equicontinuous on [0,T]. From (2.2), (2.12) and(2.13), it is easy to see that $\{S x: x \in G\}$ is uniformly bounded and equicontinuity. Since $S$ is continuous and is relatively compact, $S$ is completely continuous. By Theorem A (Kranoselskii fixed point theorem), we have a fixed point $x$ of $U+S$. That means that $x$ is a $T$-periodic solution of Eq.(1.1).

In order to prove Theorem 2.2, we need some preliminaries. Set

$$
Z:=\left\{x \mid x \in C^{1}(\mathbf{R}, \mathbf{R}), x(t+T)=x(t), \forall t \in \mathbf{R}\right\}
$$

and $x^{(0)}(t)=x(t)$ and define the norm on $Z$ as follows

$$
\|x\|=\max \left\{\max _{t \in[0, T]}|x(t)|, \max _{t \in[0, T]}\left|x^{\prime}(t)\right|\right\},
$$

and set

$$
Y:=\{y \mid y \in C(\mathbf{R}, \mathbf{R}), y(t+T)=y(t), \forall t \in \mathbf{R}\}
$$

We define the norm on $Y$ as follow $\|y\|_{0}=\max _{t \in[0, T]}|y(t)|$. Thus both $(Z,\|\cdot\|)$ and $\left(Y,\|\cdot\|_{0}\right)$ are Banach spaces.

Remark 2.5 If $x \in Z$, then it follows that $x^{(i)}(0)=x^{(i)}(T)(i=0,1)$.

Define the operators $L: Z \longrightarrow Y$ and $N: Z \longrightarrow Y$ respectively by

$$
L x(t)=x^{\prime \prime}(t), \quad t \in \mathbf{R},
$$


and

$$
\begin{aligned}
N x(t) & =-c x^{\prime \prime}(t-\tau)-a(t) x(t)+p(t) \\
& -g\left(t, x\left(t-\tau_{1}(t)\right), x\left(t-\tau_{2}(t)\right), \cdots, x\left(t-\tau_{n}(t)\right)\right), \quad t \in \mathbf{R} .
\end{aligned}
$$

Clearly,

$$
\operatorname{Ker} L=\{x \in Z: x(t)=c \in \mathbf{R}\}
$$

and

$$
\operatorname{ImL}=\left\{y \in Y: \int_{0}^{T} y(t) d t=0\right\}
$$

is closed in $Y$. Thus $L$ is a Fredholm mapping of index zero.

Let us define $P: Z \rightarrow Z$ and $Q: Y \rightarrow Y / \operatorname{Im}(L)$ respectively by

$$
P x(t)=\frac{1}{T} \int_{0}^{T} x(t) d t=x(0), \quad t \in \mathbf{R},
$$

for $x=x(t) \in X$ and

$$
Q y(t)=\frac{1}{T} \int_{0}^{T} y(t) d t, \quad t \in \mathbf{R}
$$

for $y=y(t) \in Y$. It is easy to see that $\operatorname{ImP}=\operatorname{Ker} L$ and $\operatorname{Im} L=\operatorname{Ker} Q=\operatorname{Im}(I-Q)$. It follows that $\left.L\right|_{D o m L \cap K e r P}:(I-P) Z \longrightarrow \operatorname{Im} L$ has an inverse which will be denoted by $K_{P}$.

Let $\Omega$ be an open and bounded subset of $Z$, we can easily see that $Q N(\bar{\Omega})$ is bounded and $\overline{K_{P}(I-Q) N(\bar{\Omega})}$ is compact. Thus the mapping $N$ is $L$-compact on $\bar{\Omega}$. That is, we have the following result.

Lemma 2.6. Let $L, N, P$ and $Q$ be defined by (2.17), (2.18), (2.21) and (2.22) respectively. Then $L$ is a Fredholm mapping of index zero and $N$ is $L$-compact on $\bar{\Omega}$, where $\Omega$ is any open and bounded subset of $Z$.

In order to prove Theorem 2.2, we need the following Lemma [12].

Lemma 2.7 ([12 and Remark 2.5]). Let $x(t) \in C^{(n)}(\mathbf{R}, \mathbf{R}) \cap C_{T}$. Then

$$
\left\|x^{(i)}\right\|_{0} \leq \frac{1}{2} \int_{0}^{T}\left|x^{(i+1)}(s)\right| d s, \quad i=1,2, \cdots, n-1,
$$

where $n \geq 2$ and $C_{T}:=\{x \mid x \in C(R, R), x(t+T)=x(t), \forall t \in \mathbf{R}\}$.

Now, we consider the following auxiliary equation

$$
\begin{aligned}
x^{\prime \prime}(t) \quad & +c \lambda x^{\prime \prime}(t-\tau)+a(t) \lambda x(t)=\lambda p(t) \\
& -\lambda g\left(t, x\left(t-\tau_{1}(t)\right), x\left(t-\tau_{2}(t)\right), \cdots, x\left(t-\tau_{n}(t)\right)\right),
\end{aligned}
$$

where $0<\lambda<1$.

Lemma 2.8. Suppose that conditions of Theorem 2.2 are satisfied. If $x(t)$ is a $T$-periodic 
solution of Eq.(2.23), then there are positive constants $D_{i}(i=0,1)$, which are independent of $\lambda$, such that

$$
\left\|x^{(i)}\right\|_{0} \leq D_{i}, \quad t \in[0, T], \quad i=0,1 .
$$

Proof: Suppose that $x(t)$ is a $T$-periodic solution of (2.23). We have from $\left(H_{3}\right)$ and $(2.23)$ that

$$
\left|x^{\prime \prime}(t)\right| \leq \max _{t \in[0, T]}|c|\left|x^{\prime \prime}(t)\right|+M\|x\|_{0}+\|p\|_{0}+\gamma n\|x\|_{0} .
$$

From (2.25), we have

$$
\max _{t \in[0, T]}\left|x^{\prime \prime}(t)\right| \leq \frac{1}{1-|c|}\left[(M+\gamma n)\|x\|_{0}+\|p\|_{0}\right] .
$$

On the other hand, from Lemma 2.4 and (2.23), we get

$$
\begin{aligned}
x(t) & =\int_{0}^{T} \widetilde{G}(t, s) \lambda\left[(M-a(s)) x(s)+p(s)-c x^{\prime \prime}(s-\tau)\right. \\
& -g\left(s, x\left(s-\tau_{1}(s)\right), x\left(s-\tau_{2}(s)\right), \cdots, x\left(s-\tau_{n}(s)\right)\right] d s,
\end{aligned}
$$

where

$$
\widetilde{G}(t, s)\left\{\begin{array}{c}
\widetilde{w}(t-s), \quad(k-1) T \leq s \leq t \leq k T \\
\widetilde{w}(T+t-s), \quad(k-1) T \leq t \leq s \leq k T(k \in \mathbf{N}), \\
\widetilde{w}(t)=\frac{\cos \alpha_{1}\left(t-\frac{T}{2}\right)}{2 \alpha_{1} \sin \frac{\alpha_{1} T}{2}}
\end{array}\right.
$$

$\alpha_{1}=\sqrt{\lambda M}$ and

$$
\max _{t \in[0, T]} \int_{0}^{T}|\widetilde{G}(t, s)| d s=\frac{1}{\lambda M} .
$$

From $\left(H_{3}\right),(2.27)$ and (2.30), we have

$$
\begin{aligned}
\|x\|_{0} & =\max _{t \in[0, T]} \mid \int_{0}^{T} \widetilde{G}(t, s) \lambda\left[(M-a(s)) x(s)+p(s)-c x^{\prime \prime}(s-\tau)\right. \\
& -g\left(s, x\left(s-\tau_{1}(s)\right), x\left(s-\tau_{2}(s)\right), \cdots, x\left(s-\tau_{n}(s)\right)\right] d s \mid \\
& \leq \frac{1}{M}\left[(M-m)\|x\|_{0}+\|p\|_{0}+|c| \max _{t \in[0, T]}\left|x^{\prime \prime}(t)\right|+\gamma n\|x\|_{0}\right] .
\end{aligned}
$$

From (2.31), we have

$$
\|x\|_{0} \leq \frac{|c| \max _{t \in[0, T]}\left|x^{\prime \prime}(t)\right|+\|p\|_{0}}{m-\gamma n} .
$$

Thus combining (2.26) and (2.32), we see that

$$
\max _{t \in[0, T]}\left|x^{\prime \prime}(t)\right| \leq \frac{M+m}{m(1-|c|)-M|c|-\gamma n}=\xi
$$

and

$$
\|x\|_{0} \leq \frac{|c| \xi+\|p\|_{0}}{m-\gamma n}=D_{0}
$$


Finally from Lemma 2.4, (2.33) and (2.34), we get

$$
\left\|x^{\prime}\right\|_{0} \leq D_{1} .
$$

The proof of Lemma 2.8 is complete.

Proof of Theorem 2.2: Suppose that $x(t)$ is a T-periodic solution of Eq.(2.23). By Lemma 2.8 , there exist positive constants $D_{i}(i=0,1)$ which are independent of $\lambda$ such that (2.24) is true. Consider any positive constant $\bar{D}>\max _{0 \leq i \leq 1}\left\{D_{i}\right\}+\|p\|_{0}$.

Set

$$
\Omega:=\{x \in Z:\|x\|<\bar{D}\}
$$

We know that $L$ is a Fredholm mapping of index zero and $N$ is $L$-compact on $\bar{\Omega}$ (see [3]).

Recall

$$
\operatorname{Ker}(L)=\{x \in Z: x(t)=c \in \mathbf{R}\}
$$

and the norm on $Z$ is

$$
\|x\|=\max \left\{\max _{t \in[0, T]}|x(t)|, \max _{t \in[0, T]}\left|x^{\prime}(t)\right|\right\} .
$$

Then we have

$$
x=\bar{D} \quad \text { or } \quad x=-\bar{D} \text { for } x \in \partial \Omega \cap \operatorname{Ker}(L) .
$$

From $\left(H_{3}\right)$ and (2.36), we have(if $\bar{D}$ is chosen large enough)

$$
a(t) \bar{D}+g(t, \bar{D}, \bar{D}, \cdots, \bar{D})-\|p\|_{0}>0 \text { for } t \in[0, T]
$$

and

$$
x^{\prime}(t)=0 \text { and } x^{\prime \prime}(t)=0, \text { for } t \in[0, T] .
$$

Finally from (2.18), (2.22), (2.37) and (2.38), we have

$$
\begin{aligned}
(Q N x) & =\frac{1}{T} \int_{0}^{T}\left[-c x^{\prime \prime}(t-\tau)-a(t) x(t)+p(t)\right] d t \\
& \left.-g\left(t, x\left(t-\tau_{1}(t)\right), x\left(t-\tau_{2}(t)\right), \cdots, x\left(t-\tau_{n}(t)\right)\right)\right] d t \\
& \neq 0, \quad \forall x \in \partial \Omega \cap \operatorname{Ker}(L) .
\end{aligned}
$$

Then, for any $x \in \operatorname{Ker} L \cap \partial \Omega$ and $\eta \in[0,1]$, we have

$$
\begin{aligned}
x H(x, \eta) & =-\eta x^{2}-\frac{x}{T}(1-\eta) \int_{0}^{T}\left[c x^{\prime \prime}(t-\tau)+a(t) x(t)-p(t)\right. \\
& \left.+g\left(t, x\left(t-\tau_{1}(t)\right), x\left(t-\tau_{2}(t)\right), \cdots, x\left(t-\tau_{n}(t)\right)\right) d t\right] d t \\
& \neq 0 .
\end{aligned}
$$


Thus

$$
\begin{aligned}
\operatorname{deg}\{Q N, \quad & \Omega \cap \operatorname{Ker}(L), 0\} \\
& =\operatorname{deg}\left\{-\frac{1}{T} \int_{0}^{T}\left[c x^{\prime \prime}(t-\tau)+a(t) x(t)-p(t)\right.\right. \\
& \left.\left.+g\left(t, x\left(t-\tau_{1}(t)\right), x\left(t-\tau_{2}(t)\right), \cdots, x\left(t-\tau_{n}(t)\right)\right)\right] d t, \Omega \cap \operatorname{Ker}(L), 0\right\} \\
& =\operatorname{deg}\{-x, \Omega \cap \operatorname{Ker}(L), 0\} \\
& \neq 0 .
\end{aligned}
$$

From Lemma 2.8 for any $x \in \partial \Omega \cap \operatorname{Dom}(L)$ and $\lambda \in(0,1)$ we have $L x \neq \lambda N x$. By Theorem B (Mawhin's continuation theorem), the equation $L x=N x$ has at least a solution in $\operatorname{Dom}(L) \cap \bar{\Omega}$, so there exists a T-periodic solution of Eq.(1.1). The proof is complete.

\section{References}

[1] CHEn, Y.S., The existence of periodic solutions for a class of neutral differential difference equations, Bull. Austral. Math. Soc., 33 (1992), 508-516.

[2] Chen, Y.S., The existence of periodic solutions of the equation $x^{\prime}(t)=-f(x(t), x(t-r))$, J. Math. Anal. Appl., 163 (1992), 227-237.

[3] Gaines, R.E. And Mawhin, J.L., Coincidence degree and nonlinear differential equation, Lecture Notes in Math., Vol.568, Springer-Verlag, 1977.

[4] GUo, Z.M. AND YU, J.S., Multiplicity results for periodic solutions to delay differential difference equations via critical point theory, J. Diff. Eqns., 218 (2005), 15-35.

[5] GUo, C.J. AND GUo, Z.M., Existence of multiple periodic solutions for a class of threeorder neutral differential equations, Acta. Math. Sinica, 52(4) (2009), 737-751.

[6] GUO, C.J. AND GUO, Z.M., Existence of multiple periodic solutions for a class of secondorder delay differential equations, Nonlinear Anal-B: Real World Applications, 10(5) (2009), 3825-3972.

[7] HaLE, J.K., Theory of functional differential equations, Springer-Verlag, 1977.

[8] KAPLAN, J.L. AND YoRKE, J.A., Ordinary differential equations which yield periodic solution of delay equations, J. Math. Anal. Appl., 48 (1974), 317-324.

[9] LI, J.B. AND HE, X.Z., Proof and generalization of Kaplan-Yorke's conjecture on periodic solution of differential delay equations, Sci. China(Ser.A), 42 (9) (1999), 957-964. 
[10] LI, Y.X., Positive periodic solutions of nonlinear second order ordinary differential equations, Acta Math. Sini., 45 (2002), 482-488.

[11] LU, S.P., Existence of periodic solutions for a p-Laplacian neutral functional differential equation, Nonlinear. Anal., 70 (2009), 231-243.

[12] LI, J.W. AND Wang, G.Q., Sharp inequalities for periodic functions, Applied Math. ENote, 5 (2005), 75-83.

[13] ShU, X.B., XU, Y.T. AND HuAnG, L.H., Infinite periodic solutions to a class of secondorder Sturm-Liouville neutral differential equations, Nonlinear Anal., 68 (4) (2008), 905-911.

[14] WANG, G.Q. AND YAN, J.R., Existence of periodic solutions for second order nonlinear neutral delay equations, Acta Math. Sini., 47 (2004), 379-384.

[15] XU, Y.T. AND GUo, Z.M., Applications of a $Z_{p}$ index theory to periodic solutions for a class of functional differential equations, J. Math. Anal. Appl., 257 (1) (2001), 189-205. 